\title{
Acute pulmonary edema induced by non-ionic low-osmolar radiographic contrast media
}

This article was published in the following Dove Press journal: Open Access Emergency Medicine

\section{Laurence Pincet' Gabriele Lecca ${ }^{2}$}

'ENT Surgery Department, University Hospital Centre (CHUV), Lausanne, Switzerland; ${ }^{2}$ Department of Internal Medicine, Riviera - Chablais Hospital, Monthey Site, Route de Morgins, Monthey, Switzerland
Correspondence: Laurence Pincet Centre Hospitalier Universitaire Vaudois (CHUV), Rue de Bugnon 46, I0II Lausanne, Switzerland Tel + 4I 78934240 I Email laurencepincet@gmail.com
Background: Non-cardiogenic pulmonary edema (NCPE) after intravenous (iv) administration of non-ionic radiocontrast media (RCM) is a rare but life-threatening complication. In a context of emergency, its diagnosis is difficult.

Case report: We report the case of a 55-year-old woman who developed an acute pulmonary edema following iv infusion of non-ionic, low-osmolar RCM during abdominal CT scan. She needed a 24-hour hospitalization in intensive care unit for an acute hypoxemic dyspnea. She was falsely treated at first for an anaphylactic reaction, and then for a cardiac failure. She improved with cortisone and diuretic treatment.

Conclusion: Although NCPE has been rarely reported after RCM injection, it remains an acute severe complication that has to be considered. The differential diagnosis involves multiple pathogenic patterns giving furthermore complexity in choosing an appropriate treatment. Keywords: acute pulmonary edema, non-cardiogenic pulmonary edema, radiocontrast media, adverse reaction

\section{Introduction}

Computer tomography (CT) scans are common imaging methods, and the use of contrast media exposes the patients to several risks. Contrast-induced nephropathy is the most common complication, ${ }^{1}$ and anaphylactic reaction is the most dreaded complication. However, rare cases of acute and severe respiratory distress due to noncardiogenic pulmonary edema (NCPE) after radiocontrast media (RCM) infusion have been reported. Its diagnosis is difficult, especially in a context of emergency. Given the severity of symptoms, it has to be considered as a differential diagnosis. Written informed consent was obtained from the patient for publication of this case report.

\section{Case report}

A 55-year-old woman consulted her general practitioner for a persistent abdominal pain lasting several months. An injected abdominal CT scan performed 10 months before was normal.

For a chronic effort dyspnea associated with left bundle branche, she had a previewed myocardic scintigraphy and a pulmonary function that were both normal.

Since the abdominal pain persisted, another ambulatory abdominal thoracic CT scan was performed. Non-ionic low-osmolar RCM (Iomeron $400^{\circledR}$ Bracco s.p.a., Sion, Switzerland) was injected intravenously (iv). Several seconds after the injection, the patient presented with an acute dyspnea associated with wheezing and mild hemoptysis. 
Pulmonary status showed prolonged expiratory phase and bilateral wheezing.

Suspecting an anaphylactoid reaction, she received immediately $100 \mathrm{mg}$ of hydrocortisone, $30 \mathrm{mg}$ of clemastine (iv), and $0.3 \mathrm{mg}$ of adrenaline (intramuscular), with no clinical response to this treatment. Immediately, while the patient was still lying on the CT table, a chest CT scan was performed.

She was immediately transferred to the emergency unit. At the time of admission, arterial blood gas showed $\mathrm{pH} 7.41$, arterial partial pressure of oxygen was $8.1 \mathrm{kPa}$, arterial partial pressure of carbon dioxide was $4.7 \mathrm{kPa}, \mathrm{PaO}_{2} / \mathrm{Fio}_{2}$ ratio was $18.1 \mathrm{kPa}$. Blood sample showed a mild inflammatory biologic syndrome with leukocytosis $(14.0 \mathrm{~g} / \mathrm{L})$, thrombocytosis $(401$ $\mathrm{g} / \mathrm{L})$, and slightly elevated levels of C-reactive protein (28.9 $\mathrm{mg} / \mathrm{L})$. Initially, the NT-proBNP level was around $179 \mathrm{ng} / \mathrm{L}$, and then slightly increased to $383 \mathrm{ng} / \mathrm{L}$ within 3 days. The Troponine and creatin kinase levels were in normal range. The rest of the blood test results were within normal limits, revealing no other organ failure.

The electrocardiogram revealed a block in the left bundle branch, which was already known and remain unchanged.

The thoracic CT scan revealed diffuse bilateral alveolar infiltrates and vascular redistribution, with a normal cardiac silhouette, consistent with the diagnosis of NCPE (Figure 1).

Thus, diuretic treatment was conducted in association with a high-dose corticotherapy $(1 \mathrm{mg} / \mathrm{kg} / \mathrm{d})$. She stayed in the intensive care unit for 24 hours, and no criteria for invasive ventilation was documented. After a short non-invasive ventilation therapy, oxygen has been rapidly weaned. The patient was discharged from the intensive care unit after 24 hours. No other blood arterial gas tests were conducted. The patient stayed for 48 hours in internal medicine department and then was discharged in a healthy condition.

\section{Discussion}

Severe adverse reactions, defined as requiring anesthesia or hospitalization, after the administration of low-osmolar RCM injection are rare $(0.004 \%){ }^{2}$ They are divided into immediate and delayed reactions. The onset of immediate reactions is very rapid, with about $70 \%$ occurring within 5 minutes after the injection and $96 \%$ include severe or fatal reactions such as anaphylactic shock, severe angioedema, pulmonary edema, and cardiac arrest, occurring within 20 minutes after injection. ${ }^{3}$

Pulmonary edema is reported to be a rare $(0.001 \%-$ $0.008 \%$ ) adverse reaction to iv injection of any type of contrast media. However, it constitutes $10 \%-20 \%$ of lethal reactions developing as a result of iv infusion of contrast media. ${ }^{4}$ Therefore, physiopathology of this condition needs to be understood.

Two types of pulmonary edema are recognized: cardiogenic and non-cardiogenic. Non-cardiogenic edema involves an increase of microvascular permeability, thus leading to alveolar edema. In the context of RCM injection, each of them can be explained as follows:

\section{Cardiogenic}

Pulmonary edema can partly be related to a cardiac failure, since this condition can be induced by several mechanisms.

Contrast media can be grouped as high- or low-osmolar agents. High-osmolar contrast media are not used anymore. The normally used low-osmolar contrast media are classified, according to their viscosity at $20^{\circ} \mathrm{C}$, as high- or low-viscosity contrast agents.
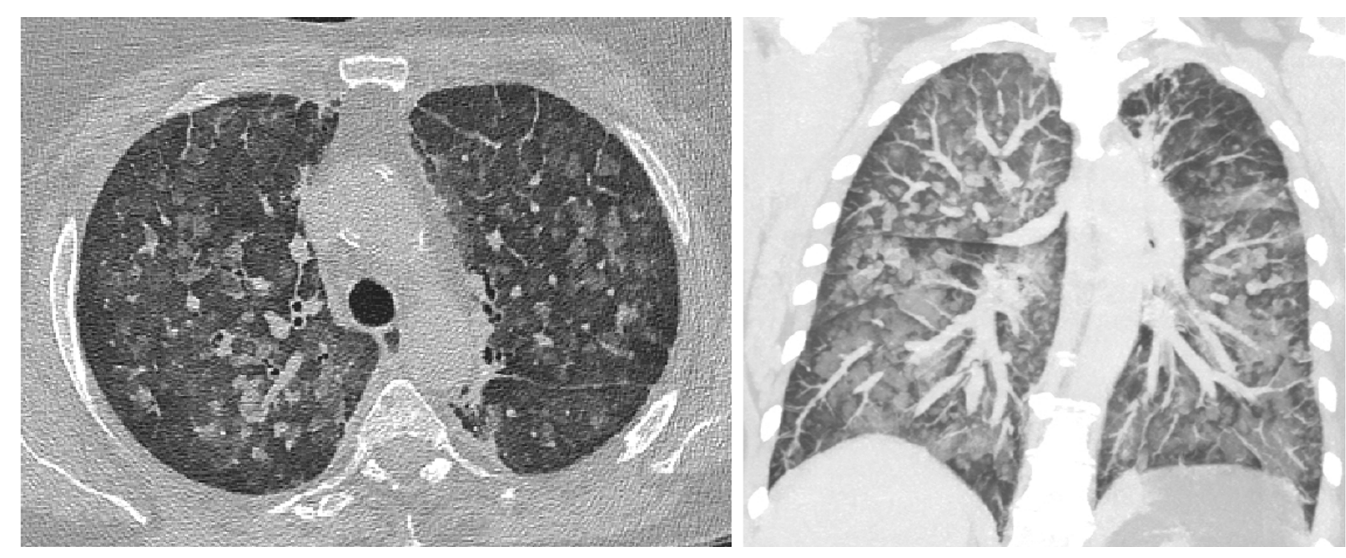

Figure I Thoracic CT-scan, $7 \mathrm{~mm}$ thick slices.

Note: It shows a pattern of ground-glass appearance, without clear dependent location. 
Even the low-osmolar and less viscous media show higher osmolarity and are more viscous than blood at $37^{\circ} \mathrm{C}$ (Figure 2). Poiseuille's law states that viscosity is inversely related to flow (Figure 3). Thus, injection of the contrast media (at $20^{\circ} \mathrm{C}$ ) into the venous blood $\left(\right.$ at $37^{\circ} \mathrm{C}$ ) under high pressure generates a new resistance, and involves a higher cardiac effort to maintain the same ejection fraction. Without cardiac adaptation, this will cause a cardiac overload, leading to a cardiac failure and pulmonary edema.

It is known from the Stokes-Einstein equation (Figure 4) that viscosity is related to temperature. Kerl et al showed that the use of warmer contrast media could be beneficial because higher temperatures lower contrast media viscosity. ${ }^{5}$

This process needs some time to develop and cardiogenic high pressure gives a particular pattern images in radiology of peri-hilar ground-glass.

\section{Non-cardiogenic}

Various other reasons behind the non-cardiogenic mechanisms of pulmonary edema have been studied. One of them is the endothelial injury. Experimental studies have shown that mediator release and complement activation result in endothelial damage. ${ }^{6,7}$ Following the Starling relationship (Figure 5), we can easily understand that an increase in capillary wall's permeability leads to an accumulation of fluid in the lung.

At the cellular level, it has been shown that iodinated contrast media influences erythrocyte morphology, which is partially related to contrast media osmolarity. ${ }^{5} \mathrm{~A}$ highosmolar contrast media causes red blood cells dehydration, observed in vitro as dessicocytes. ${ }^{8}$ The use of iso-osmolar contrast media, rather than low-osmolar contrast media, would result in less damage to the red blood cells.

\begin{tabular}{|l|l|}
\hline$Q=\triangle P R 4 \pi / 8 \eta L$ \\
\hline$Q:$ rate of flow \\
$\Delta P:$ pressure gradient \\
$R \quad:$ radius of tube \\
$\eta \quad:$ viscosity of fluid \\
$L \quad:$ length of tube \\
\hline
\end{tabular}

Figure 3 Poiseuille's law of flow.

Notes: Poiseuille Law states that the flow $(Q)$ of fluid is related to a number of factor: the viscosity $(\eta)$ of the fluid, the pressure gradient across the tubing $(\Delta p)$, and the lenght $(\mathrm{L})$ and diameter $(r)$ of the tubing. It can be successfully applied to air flow in lung alveoli.

\begin{tabular}{|l|}
\hline \multicolumn{1}{|c|}{$\eta=(K 1 \times e K 2 / T) \times F \times 1 / s \times V$} \\
$V:$ dynamic viscosity \\
$F:$ resistance \\
$I:$ distance \\
$K 1$ and $K 2:$ constants related to the temperature \\
$T:$ temperature
\end{tabular}

Figure 4 Stokes-Einstein equation.

Note: Viscosity is inversely related to temperature.

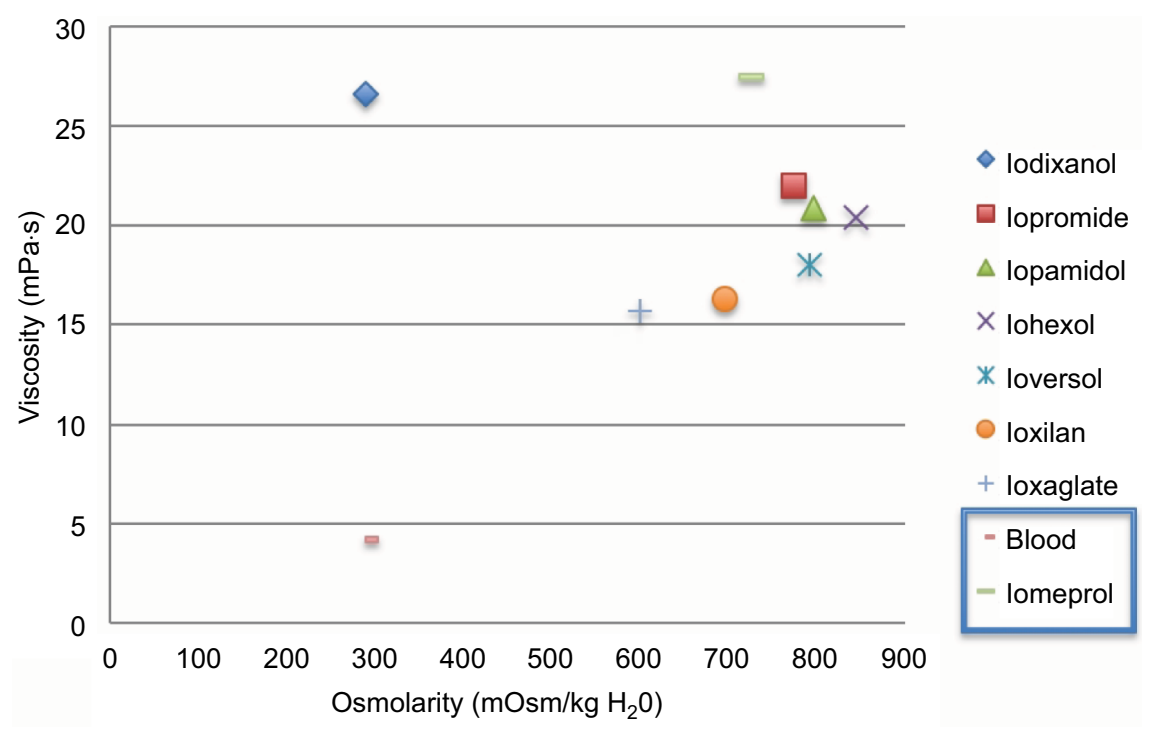

Figure 2 Differences in viscosity and osmolarity between several non-ionic low-osmolar contrast media at $20^{\circ} \mathrm{C}$ and blood at $37^{\circ} \mathrm{C}$. 
Net filtration $=(L p \times S) \times(\Delta$ hydraulic pressure $-\Delta$ oncotic pressure $)$ $=($ Lp $\times S) \times(($ Pcap - Pif $)-S(\pi c a p-\pi$ if $))$

Lp : permeability of the capillary wall

$S$ : Surface area available for fluid movement

Pcap and Pif : capillary and interstitial fluid hydraulic pressures

$\pi$ cap and $\pi$ if : capillary and interestital fluid oncotic pressures

$S$ represents the reflexion coefficient of proteins across the capillary wall

Figure 5 The Starling relationship.

Notes: Starling's law of the capillaries states that the movement of fluid between the capillaries and interstitial fluid is due to the net effect of all four of the pressures described.

Moreover, damaged red cells are more rigid, causing precapillary stasis ${ }^{8}$ and an increase of blood viscosity, leading to a cardiac failure and pulmonary edema, as stated earlier. This toxic mechanism is immediate and is identified in radiology by a mosaic pattern of ground-glass appearance.

\section{Our case}

The two major differential diagnoses for this acute dyspnea developed after the injection of contrast media were anaphylactic reaction or acute pulmonary edema. In this critical situation, it was difficult to exclude both of these life-threatening diagnoses. The first care were given in the emergency unit, before differential diagnosis could clearly be ruled out. The diagnostic of NCPE was then established.

The sudden onset of symptoms indicated a cardiogenic mechanism. However, a recent adenosine-stressed myocardial perfusion scintigraphy showed a good cardiac function. The NT-proBNP rate was initially in the normal range, and there was no electrical or biological indication for a cardiac ischemia. The CT image showed a pattern of ground-glass appearance, without clear dependent location, suggesting a NCPE.

However, we cannot exclude a small implication of cardiogenic mechanism. The patient had a history of unexplained chronic effort dyspnea. The contrast media used was highly viscous with high osmolarity (Figure 2). Secondary, slight elevation of the NT-proBNP rate is compatible with a cardiac overload. However, it is not obvious if cardiac overload is secondary to contrast media or to NCPE.

\section{Treatment}

For a case of fatal NCPE resulting after iv infusion of nonionic RCM, Paul and George recommended treatment by administration of oxygen with continuous positive airway pressure or invasive ventilation, with positive end expiratory pressure. ${ }^{7}$ Diuretics or vasodilators should be avoided.
Moreover, they recommend a fluid resuscitation to increase left ventricular preload.

In our case, immediate treatment with hydrocortisone, clemastine, and adrenaline had no effect in the beginning. The continuation of cortisone in association with diuretic showed a good response. However, this improvement seems paradoxical. On one hand, cortisone may have reduced the inflammatory response and endothelial injury; on the other hand, diuretics could have treated the cardiac overload component. Indeed, NPCE can cause an increase of vascular resistance, leading to a change in the preload and therefore to a secondary cardiac failure.

\section{Conclusion}

In medical practice, especially in an emergency context, the dichotomy between NCPE and CPE, represent a burden in diagnostic andare treatment process. Indeed it requires choose between giving fluids instead diuretic therapy. Moreover, they may overlap. Therefore, it (the choice) must be driven by the practitioner estimation.

Knowledge of the patient's medical history including pre-existing cardiovascular compromise, sudden onset of the dyspnea symptoms, laboratory tests with NT-proBNP, and eventually chest CT scan can help to distinguish one condition from the other. In our case, treatment with cortisone and diuretics led to symptom resolution. However, it was not the adequate treatment for $\mathrm{NCPE}$, and the outcome could have been worse.

\section{Learning points}

1. The existence of prior CT scans cannot be totally reassuring because life-threatening adverse reactions can occur without involving any anaphylactoid mechanism.

2. Osmolarity and viscosity of contrast media seem to play a major role in life-threatening adverse reactions and low-osmolar contrast media with less viscosity should be preferred.

3. As well, comparison of the effect of contrast media heated to $37^{\circ} \mathrm{C}$ versus contrast media at ambient temperature could be interesting in order to decreased the risk, at minimum cost.

\section{Author contributions}

All authors contributed toward data analysis, drafting and critically revising the paper and agree to be accountable for all aspects of the work.

\section{Disclosure}

The authors report no conflicts of interest in this work. 


\section{References}

1. Andreucci M, Solomon R, Tasanarong A. Side effects of radiographixy contrast media: pathogenesis, risk factors, and prevention. Biomed Res Int. 2014;2014:741018.

2. Voeltz MD, Nelson MA, Mc Daniel MC, et al. The important properties of contrast media: focus on viscosity. J Invasive Cardiol. 2007;19:1A-9A.

3. Kang MH, Nah JC. A delayed, unusual non cardiogenic pulmonary edema after intravascular administration of non-ionic, low osmolar radiocontrast media for coronary angiography. Korean Circ J. 2013;43:500-503.

4. Hauggard A. Non-cardiogenic pulmonary oedema after intravenous administration of non-ionic contrast media. Acta Radiol. 1996;37:823-825.
5. Kerl JM, Nguyen SA, Lazarchick J, et al. Iodinated contrast media: effect of osmolarity and injection temperature on erythrocytes morphology in vitro. Acta Radiol. 2008;49:337-343.

6. Morcos SK. Pulmonary effects of radiographic contrast media. In: Thomsen HS, editor. Contrast Media: Safety Issues and ESUR Guidelines. Sheffield, UK: Northern General Hospital NHS Trust; 2006:83-88.

7. Paul RE, George G. Fatal non-cardiogenic pulmonary oedema after intravenous nonionic radiographic contrast. Lancet. 2002;359:1037-1038.

8. Aspelin P, Stacul F, Morcos SK. Effects on the blood and endothelium. In: Thomsen HS, editor. Contrast Media: Safety Issues and ESUR Guidelines. Karolinska Institute, Huddinge University Hospital; 2006: 65-74.
Open Access Emergency Medicine

\section{Publish your work in this journal}

The Open Access Emergency Medicine is an international, peerreviewed, open access journal publishing original research, reports, editorials, reviews and commentaries on all aspects of emergency medicine. The manuscript management system is completely online and includes a very quick and fair peer-review system, which is all

\section{Dovepress}

easy to use. Visit http://www.dovepress.com/testimonials.php to read real quotes from published authors. 